ARTICLE

https://doi.org/10.1038/s41467-019-13730-0

\title{
Relaxed purifying selection in autopolyploids drives transposable element over-accumulation which provides variants for local adaptation
}

Pierre Baduel1', Leandro Quadrana1', Ben Hunter², Kirsten Bomblies ${ }^{3}$ \& Vincent Colot (1) ${ }^{1 \star}$

Polyploidization is frequently associated with increased transposable element (TE) content. However, what drives TE dynamics following whole genome duplication (WGD) and the evolutionary implications remain unclear. Here, we leverage whole-genome resequencing data available for $\sim 300$ individuals of Arabidopsis arenosa, a well characterized natural diploidautotetraploid plant species, to address these questions. Based on 43,176 TE insertions we detect in these genomes, we demonstrate that relaxed purifying selection rather than transposition bursts is the main driver of TE over-accumulation after WGD. Furthermore, the increased pool of TE insertions in tetraploids is especially enriched within or near environmentally responsive genes. Notably, we show that the major flowering-time repressor gene FLC is disrupted by a TE insertion specifically in the rapid-cycling tetraploid lineage that colonized mainland railways. Together, our findings indicate that tetrasomy leads to an enhanced accumulation of genic TE insertions, some of which likely contribute to local adaptation.

\footnotetext{
${ }^{1}$ Institut de Biologie de I'Ecole Normale Supérieure (IBENS), Centre National de la Recherche Scientifique (CNRS), Institut National de la Santé et de la Recherche Médicale (INSERM), Ecole Normale Supérieure, PSL Research University, 75005 Paris, France. ${ }^{2}$ Department of Organismic and Evolutionary Biology, Harvard University, Cambridge, MA 02138, USA. ${ }^{3}$ ETH Zürich, 8092 Zürich, Switzerland. *email: colot@biologie.ens.fr
} 
E ukaryotic genomes display remarkable variation in ploidy as well as in size. This is particularly true of plant genomes, the evolution of which has been punctuated by numerous whole genome duplication (WGD) events ${ }^{1-3}$. Moreover, polyploidization is often associated with an increase in transposable element (TE) content, which further exacerbates the inflation of genome size $e^{3,4}$. This association has long been seen as supporting the "genome-shock" hypothesis initially proposed by Barbara McClintock ${ }^{5}$, where genomic instabilities associated with genome doubling could trigger transposition bursts. However, the possibility of such bursts has been studied primarily in allopolyploids, where the effects of WGD and hybridization are confounded (e.g. refs. ${ }^{6,7}$ ). Furthermore, the doubling of genome copies should double the mutation rate per individual and reduce the selective pressure exerted on recessive deleterious mutations ${ }^{8}$, especially in autopolyploids where all homologous chromosomes segregate randomly (polysomic masking). Therefore, the loss-of-function mutations typically caused by TE insertions could readily overaccumulate in polyploids, even in the absence of transposition bursts.

These two non-mutually exclusive scenarios to account for the increased TE content of polyploids are still presented indiscriminately (e.g. ref. ${ }^{9}$ ) because of a lack of experimental support for one or the other ${ }^{10,11}$. Yet, their evolutionary implications differ substantially: while transposition bursts resulting from the WGD event are expected to impact the fitness of neo-polyploids in the first few generations after they are formed ${ }^{12,13}$, the effects of relaxed purifying selection due to polysomic masking would only be perceived many generations downstream, as a result of the progressive accumulation of new TE insertions. In any case, the slower allele dynamics caused by relaxed purifying selection in polyploids ${ }^{14}$ do not necessarily imply slower rates of adaptation. Indeed, when environments change, previously deleterious alleles can turn beneficial. Thus, the increased amount of standing variation in polyploids is expected to result in faster adaptive responses $^{15}$. Moreover, polyploids may also benefit directly from their higher mutation rate per individual, if some mutations are at least partially dominant ${ }^{15}$.

Here, we set out to determine the respective importance of the two possible routes to TE accumulation following WGD using Arabidopsis arenosa as a model system. This plant species occurs in both diploid and autotetraploid forms across Central and Northern Europe ${ }^{16}$. The autotetraploids are fully tetrasomic and originated in the Western Carpathians from a single recent WGD event $^{16}$ ( $60 \mathrm{kyr}$ ago). They rapidly expanded and now occupy a broader ecological range than their diploid progenitors ${ }^{17}$. Notably, as frequently reported for polyploids ${ }^{18,19}$, one $A$. arenosa tetraploid lineage successfully invaded a novel ruderal habitat, where they adopted a weedy life-cycle not shared by diploids or non-ruderal tetraploid $A$. arenos $a^{20,21}$. This notwithstanding, the largely overlapping ecological niches and the recent divergence between the two $A$. arenosa lineages allows for cross-ploidy genomic comparisons ${ }^{22}$.

Using the most comprehensive diploid-autopolyploid dataset to date $\mathrm{e}^{22}$, we characterize TE dynamics across diploid and tetraploid $A$. arenosa genomes. First, we assess how the relaxation of purifying selection impacted TE dynamics in autotetraploids. Second, we investigate whether a transposition burst was associated with the WGD event that all $A$. arenosa tetraploids trace back to. Finally, we ask whether and how TE dynamics in autotetraploids might contribute to their adaptive potential.

\section{Results}

TE landscapes in diploid and tetraploid $A$. arenosa genomes. Genome resequencing data are available for 105 diploid and 181 tetraploid individuals of $A$. arenosa, corresponding to 15 and 23 distinct populations covering the entire range of the species, respectively ${ }^{22}$. In the absence of a reference $A$. arenosa genome sequence, we used that of the closely related Arabidopsis lyrata species $^{23}$ as was done in previous SNP-based population genomic studies of $A$. arenosa $a^{16,22,24}$

We assessed recent $\mathrm{TE}$ activity in $A$. arenosa using the SPLITREADER pipeline ${ }^{25}$ (see Methods), which enabled us to identify 43,176 non-reference TE insertions (hereafter simply designated as TE insertions) from split and discordant reads in the 286 re-sequenced $A$. arenosa genomes aligned on the A. lyrata reference genome. These TE insertions correspond to three class I (LINE, Copia, and Gypsy retrotransposons) and five class II (Mariner, MuDR, hAT, Harbinger, and CACTA DNA transposons) superfamilies, in proportions similar to those found in $A$. lyrata $^{26}$ (Fig. 1a). However, unlike annotated TE sequences of the A. lyrata reference genome, which are heavily enriched towards pericentromeric regions, the TE insertions we detected are homogeneously distributed along chromosomes with no obvious pericentromeric bias (Fig. 1b).

Purifying selection shapes TE landscapes in diploids. To characterize more precisely TE localization and dynamics we started with the 105 diploid (i.e. $2 \times$ ) individuals. The density of TE insertions is higher on the chromosome arms than within $5 \mathrm{Mb}$ of the centromeres and follows that of the genes rather than the TEs annotated in the A. lyrata reference genome (Fig. 1c). In order to test whether this higher density of TE insertions within chromosome arms reflects recent TE activity or detection biases against pericentromeric regions, we compared the distribution along chromosomes of those TEs at low- and high- carrier frequencies (2x-LF vs 2x-HF, see Methods). This parameter is a proxy for relative age when positive selection is rare, because recent TE insertions are unlikely to be shared by more individuals than older ones. Across all TE superfamilies, we observed a clear and consistent decrease $\left(-13.5 \%, \chi^{2}\right.$-test $\left.p<0.001\right)$ in the density and proportion $\left(p_{\mathrm{arm}}\right)$ of HF compared to LF TE insertions within chromosome arms (Fig. 1d, e). Furthermore, outside of pericentromeric regions HF TE insertions were found significantly less frequently than LF TE insertions in gene-rich compared to genepoor regions $\left(\chi^{2}\right.$-test $p$-value $<1 \mathrm{E}-7$, Table S1). This distribution shift was also associated with a deficit of HF TE insertions within genes, specifically within exons and introns (Fig. 1f), as well as near $(<250 \mathrm{bp})$ genes (Fig. 1g). These observations are consistent with purifying selection acting to progressively filter out TE insertions from chromosome arms due to their generally deleterious effects on genes, as reported in $A$. thaliana ${ }^{25}$. In keeping with this interpretation, we found a significant association between changes in transcript levels and presence of TE insertions within or near $(<250 \mathrm{bp})$ genes but not when further away (Supplementary Fig. 1a, b, see Methods). These effects, mostly negative, likely reflect the impact of TE insertions on the expression of nearby genes but could also be the result of insertion preferences for haplotypes already differentially expressed.

Further analysis revealed that the deficit of HF TE insertions within or near genes is most pronounced for the Copia, Gypsy, CACTA, and hAT TE superfamilies, which show an apparent insertion preference (evaluated using LF TE insertions) for genes, especially exons. Specifically, the proportion of these TE insertions that lie within exons and introns are much reduced ( $-20.0 \%$ and $-4.2 \%$, respectively) at high frequency (Fig. 1f), consistent with them having the most detrimental effects and therefore being the most rapidly purged by natural selection. TE insertions from these superfamilies are hereafter called "type A" (Fig. 1h and Supplementary Fig. 1c). 
a
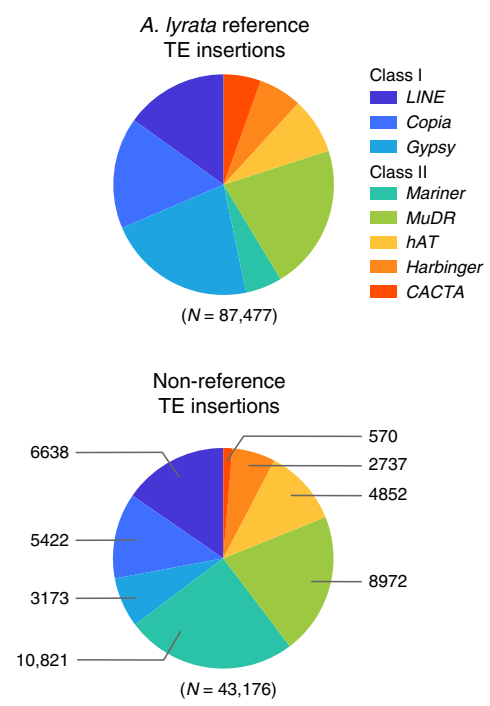

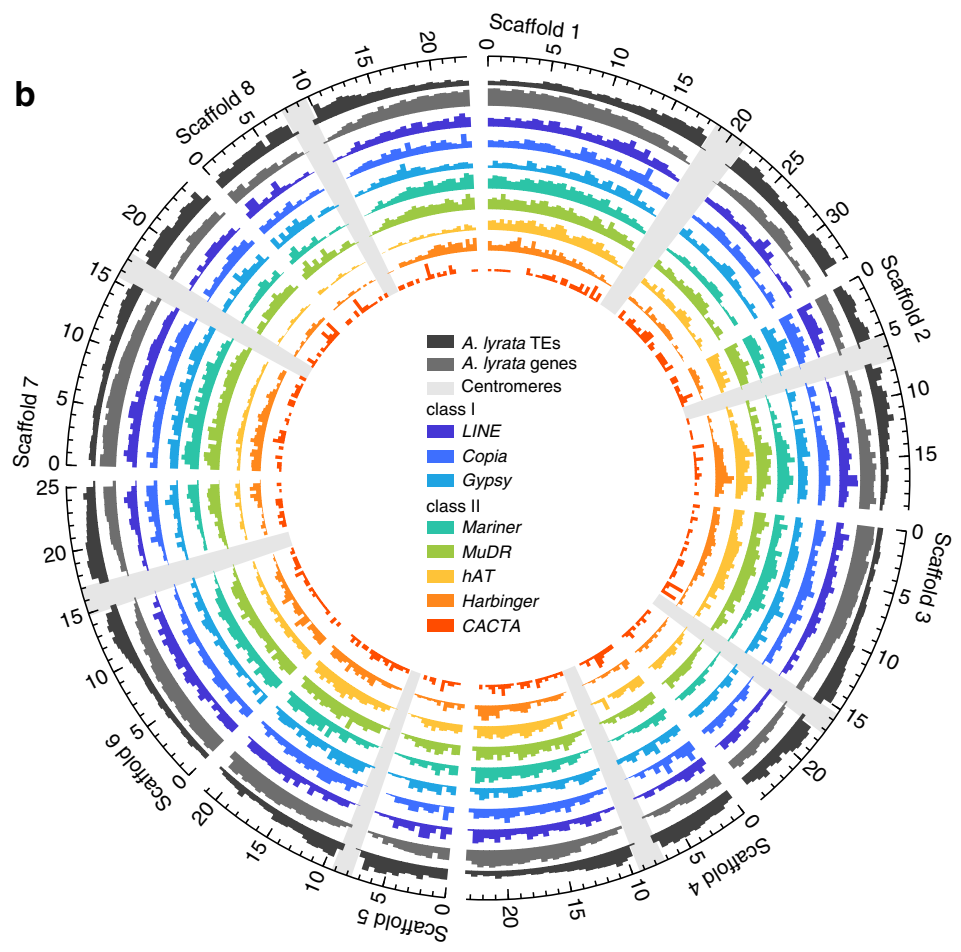

d

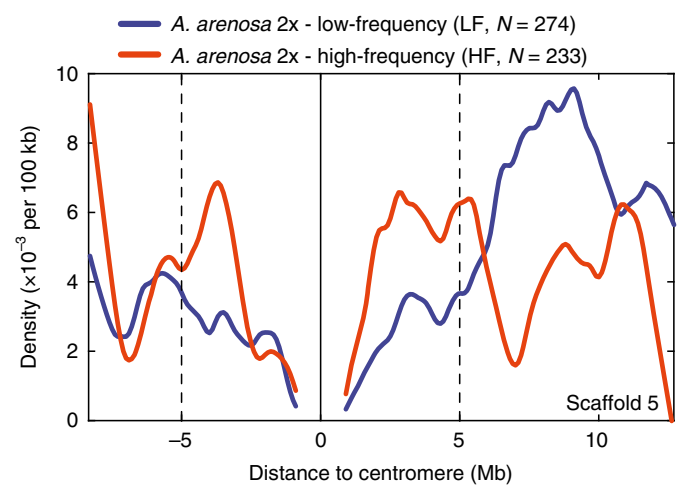

g

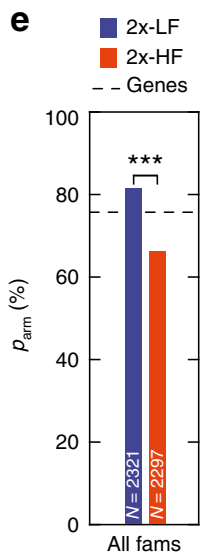

f

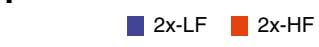

Type A

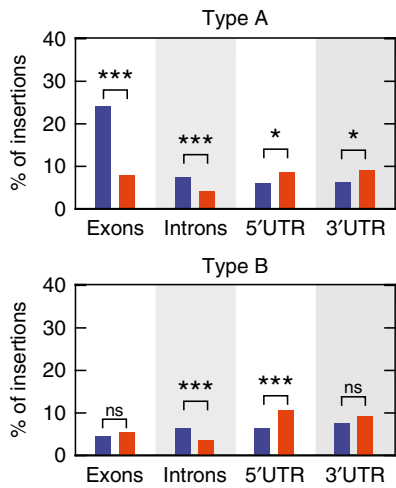

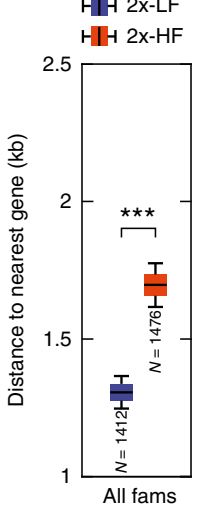

h
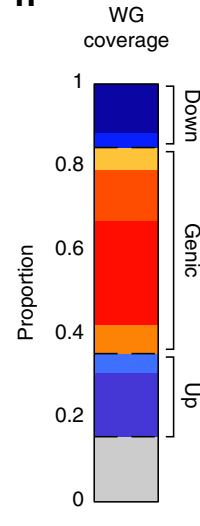
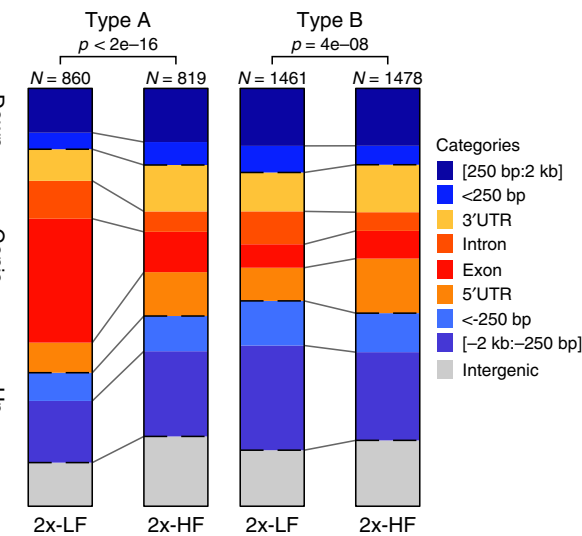

In contrast, HF as well as LF TE insertions from the other TE superfamilies (LINE, Mariner, MuDR, and Harbinger), thereafter called "type B", are underrepresented within exons (Fig. 1f-h and Supplementary Fig. 1c). It is not clear whether this observation reflects insertion preferences away from exons or stronger and more dominant deleterious effects of type $B$ insertions within exons, which would thus be rapidly purged and therefore rarely detected even at low frequency.

The deficit of HF TE insertions within exons (type A) or introns (types $\mathrm{A} \& \mathrm{~B}$ ) is not compensated uniformly across other categories. Rather, the increased proportion of HF TE insertions was most pronounced in the $5^{\prime}$ or $3^{\prime}$ UTRs of genes for both types 
Fig. 1 Natural selection shapes the TE landscape of A. arenosa diploids. a Distribution of reference TEs (upper chart) and non-reference (lower chart) TE insertions identified by SPLITREADER across the 8 class I \& II TE superfamilies. b Chromosomal distributions of reference genes and TEs and of nonreference TE insertions by TE superfamily across the 8 scaffolds of the A. lyrata reference genome. $\mathbf{c}$ Density per $100 \mathrm{~kb}$ of reference genes and TEs and of non-reference diploid TE insertions across scaffold 5. d Density per $100 \mathrm{~kb}$ across scaffold 5 of low- and high-frequency TE insertions in diploids. e Fraction, $p_{\text {arm, }}$ within chromosome arms ( $>5 \mathrm{Mb}$ away from centromeres) of low- and high-frequency TE insertions in diploids with $p$-values of $\chi^{2}$-test. ( $f$ ) Fraction of low- and high-frequency TE insertions in diploids overlapping exons, introns, $5^{\prime}$ or $3^{\prime}$ UTRs for type A (upper panel, $n=8602 x-L F$ and $n=8192 x-\mathrm{HF}$ ) and type $B$ (lower panel, $n=14612 x-\mathrm{LF}$ and $n=14782 x-\mathrm{HF}$ ) superfamilies with $p$-values of $\chi^{2}$-test. $\mathbf{g}$ Boxplots of distance to nearest gene (kb) of low- and high-frequency non-genic TE insertions in diploids across 1000 bootstraps and $p$-value of $t$-test between ploidies. Boxplot center lines, median; box limits, upper and lower quartiles; whiskers, 9th and 91st quantiles. $\mathbf{h}$ Distribution of low- and high-frequency TE insertions in diploids across categories of insertions for type $A$ and type B superfamilies compared to reference genome annotations with $p$-values of $\chi^{2}$-test. $p<0.001:{ }^{\star \star \star} ; p<0.01:{ }^{\star \star} ; p<0.05:$; $p \geq 0.05$ : ns. The source data underlying Fig. $1 \mathrm{~b}-\mathrm{h}$ are provided as a Source Data file.

of TE superfamilies $(+4.5 \%$ and $+2.6 \%$ respectively, Fig. 1f and Supplementary Fig. 1c). Although we cannot rule out alternative hypotheses, this observation suggests that TE insertions located within these two particular genic compartments tend to be under positive rather than purifying selection.

Polysomic masking causes TE over-accumulation in tetraploids. In contrast to diploids, autotetraploids (i.e. $4 \mathrm{x}$ ) show a marked increase in the proportion $\left(p_{\text {exon }}\right)$ of type A insertions within exons $\left(\chi^{2}\right.$-test $p<0.001$, Fig. $2 a, b$ and Supplementary Fig. 2a). In addition, type A insertions are slightly more frequent within chromosome arms $\left(\chi^{2}\right.$-test $p<0.05$, Fig. $2 c$ and Supplementary Fig. $2 \mathrm{~b})$ and closer to genes $(0.10 \mathrm{~kb}, t$-test $p<0.001$, Fig. 2d and Supplementary Fig. 2c) in the autotetraploids relative to diploids. The proportion ( $p_{\text {UTRs }}$ ) of type A insertions within UTRs are mostly unaffected by ploidy (Fig. 2e). In contrast, type $\mathrm{B}$ insertions showed few differences between ploidies, except for a small increase in their proportion within UTRs in the tetraploids (Fig. 2e), the significance of which is unclear. Thus, outside of type A exonic insertions, differences between ploidies remain limited. Moreover, the overall carrier-frequency spectra did not significantly differ (Supplementary Fig. 3a) despite potential biases affecting differently the detection of TE insertions in autotetraploids (see Methods). Combined, these observations confirm that the TE landscapes captured in diploids and tetraploids are globally similar, in line with the relatively recent origin of the $A$. arenosa autotetraploids ${ }^{16}$, and are consistent with purifying selection being significantly relaxed on genic TE insertions in the autotetraploids.

In keeping with this scenario, when corrected for coverage per haploid genome (haplo-coverage) tetraploids tend to harbor a higher number of TE insertions than their diploid counterparts (see Methods, Fig. 2f, Supplementary Table 2) and this increase is predominantly contributed by exonic TE insertions. Furthermore, tetraploids on average carry $\sim 30 \%$ more low-frequency TE insertions (mostly type A) within exons than diploids (see Methods), but the number of high-frequency TE insertions is similar between diploids and autotetraploids (Fig. $2 \mathrm{~g}$ and Supplementary Fig. 2d). Given that autotetraploids arose relatively recently ${ }^{16}$ and are therefore unlikely to have reached their mutational balance equilibrium ${ }^{22}$, we can indeed expect the relaxation of purifying selection to be mostly visible over lowfrequency TE insertions, as these tend to be young and not shared with diploids.

According to the genome-shock hypothesis ${ }^{9}$, the global increase in TE content observed in the autotetraploids could also be the footprint of a general transposition burst at the time of the WGD event. Under this scenario, tetraploids compared to diploids should exhibit an excess of high-frequency TE insertions, especially when non-genic, as these are the least exposed to purifying selection (Fig. 1h). This is clearly not the case, as tetraploids carried less non-genic high-frequency TE insertions than diploids, but similar amounts in genes (Fig. 3a, see Methods). After correction for haplo-coverage, we observed an excess of non-genic TE insertions in the tetraploids at lowfrequency only (Fig. 3b, Supplementary Table 3), all but ruling out an ancestral genome-wide transposition burst. Nonetheless, $35 \%$ of high-frequency non-genic TE insertions in the autotetraploids are not present in the diploids (Supplementary Fig. 3b), suggesting that they could have originated from TEfamily-specific transposition bursts. To explore this possibility, we compared the proportion of high-frequency non-genic TE insertions between tetraploids and diploids for the $201 \mathrm{TE}$ families with enough non-reference copies $(>10)$ in each of the two ploidy groups and found that no TE family shows a significant excess $\left(\chi^{2}, p<0.05\right)$ of high-frequency non-genic TE insertions in the autotetraploids, with one possible exception (ALLINE1_2.1; $p=0.036$, Fig. 3c).

Collectively, our data indicate that the increased TE content in autotetraploids results mainly if not exclusively from the progressive accumulation of TE insertions within genes, especially exons, thanks to relaxed purifying selection, rather than from any appreciable transposition burst.

Copia over-accumulation offers variants for local adaptation. Given the association of polyploidy with colonization potential ${ }^{27-}$ ${ }^{29}$, we asked whether the increased TE content within or near genes could contribute to local adaptation of autotetraploids. To this end, we first identified clade-specific TE insertions for the two ploidy groups (See Methods, Fig. 4a) and found a significant enrichment in tetraploids compared to diploids within or near $(<250 \mathrm{bp})$ genes, which is most pronounced for local high-frequency type A insertions (Fig. 4b and Supplementary Fig. 4a). This finding suggests therefore that type A insertions within or near genes are commonly under local positive selection in tetraploids, unlike those of type B (Fig. 4b). Further analysis of these local high-frequency type A insertions indicated that they are specifically enriched for Copia LTR-retrotransposons in tetraploids (Fig. 4c). Consistent with observations in $A$. thaliana and other plant species ${ }^{30}$, this TE superfamily shows in both ploidies a marked insertion preference, visible at low-frequency, towards genes responsive to biotic stimuli (Supplementary Fig. 4b), a class of genes that are typically under diversifying selection ${ }^{31,32}$. Moreover, Copia insertions within or near such genes are significantly enriched at local high-frequency only in tetraploids (Fig. 4d) and are more likely to reach high frequencies (three or more individuals within a clade, HF3) than all other TE insertions (Fig. 4e), independently of the longer size of this category of genes (Supplementary Fig. 4c). Collectively these findings indicate that Copia insertions within environmentally responsive genes are more frequently under local positive selection specifically in tetraploids. 
a
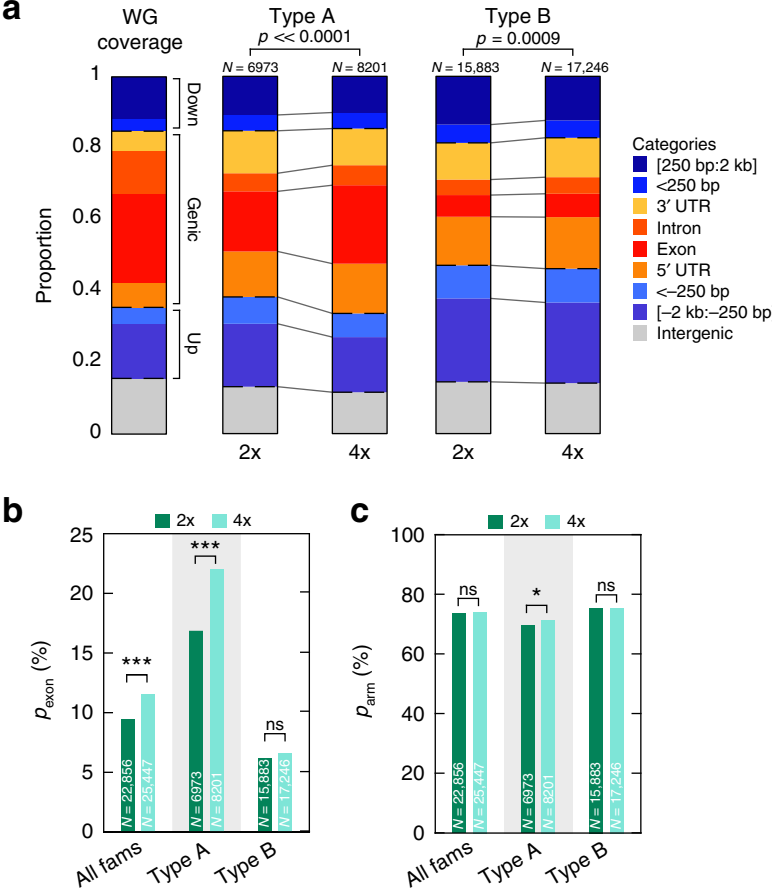

C

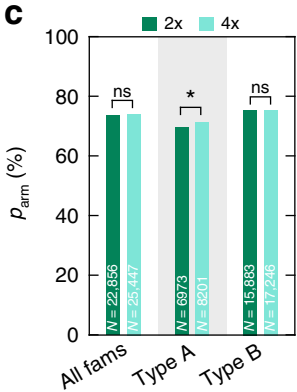

d

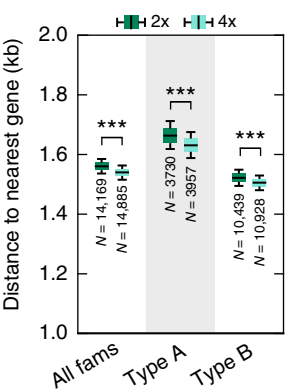

e
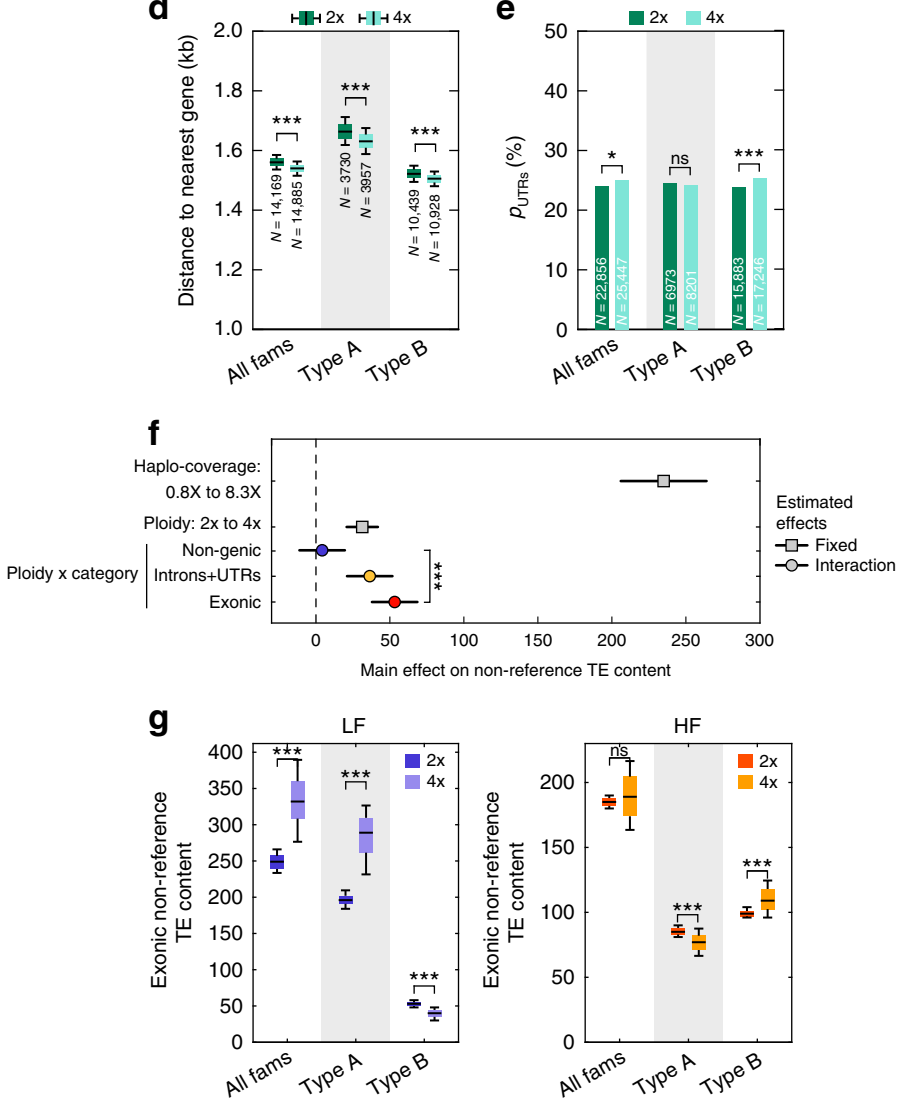

One clear example of local adaptation of $A$. arenosa tetraploids to a novel habitat is provided by the successful colonization by one lineage of railway ballasts (Fig. 5a) across central Europe. In previous studies, we have shown that adaptation to the harsher railway environment is associated with a switch to early flowering and a loss of expression of the floral repressor gene $F L C^{20,21}$ (Fig. 5b, c). FLC is a known hotspot for Copia insertions in $A$. thaliana ${ }^{25}$, so we investigated whether railway tetraploids may be

carrying such an insertion. Due to local syntenic divergences between $A$. arenosa and $A$. lyrata in the FLC region ${ }^{33}$, we could not use our $A$. lyrata-based pipeline to search for the presence of non-reference TE insertions within the two full length $A$. arenosa FLC paralogues (AaFLC1 \& AaFLC2). Instead, we sequenced three fosmid clones of the $F L C$ region that were obtained from an early flowering railway individual. Comparison to the $\mathrm{BAC}$ sequence of the FLC region that was obtained previously from a 
Fig. 2 Increased exonic TE load from relaxed purifying selection in autotetraploids. a Distribution of TE insertions across categories of insertions for type $\mathrm{A}$ and type $\mathrm{B}$ superfamilies in diploids and tetraploids compared to reference genome annotations with $p$-values of $\chi^{2}$-test. $\mathbf{b}$ Fraction, $p_{\text {exon, of TE insertions }}$ overlapping exons for all, type A, and type B superfamilies in diploids and tetraploids with $p$-values of $\chi^{2}$-test. $\mathbf{c}$ Fraction, $p_{\text {arm }}$, within chromosome arms ( $>5 \mathrm{Mb}$ away from centromeres) of TE insertions for all, type $\mathrm{A}$, and type B superfamilies in diploids and tetraploids with $p$-values of $\chi^{2}$-test. $\mathbf{d}$ Boxplots of distance to nearest gene of non-genic TE insertions for all, type A, and type B superfamilies in diploids and tetraploids across 1000 bootstraps and $p$-value of $t$-test between ploidies. Boxplot center lines, median; box limits, upper and lower quartiles; whiskers, 9th and 91st quantiles. e Fraction, $p_{U T R s,}$ of TE insertions overlapping UTRs ( $5^{\prime}$ and $3^{\prime}$ ) for all superfamilies, type A, and type B TE superfamilies in diploids and tetraploids with $p$-values of $\chi^{2}$-test. f Estimated MLM effects and interaction terms of haplo-coverage, ploidy, and category of insertions (non-genic, introns and UTRs, or exonic) on nonreference TE content. Horizontal lines indicate $95 \%$ confidence intervals for each effect value. $p$-values for each coefficient corresponds to the $t$-statistic of the hypothesis test that the corresponding coefficient is equal to zero or not. $\mathbf{g}$ Number of TE insertions within exons carried by 100 individuals for all, type $A$, and type B superfamilies in diploids and tetraploids at low-frequency (LF, left panel) and high-frequency (HF, right panel) with standard deviations across 100 random samples and $p$-value of $t$-test between ploidies. Boxplot center lines, median; box limits, upper and lower quartiles; whiskers, 9th and 91st quantiles. $p<0.001$ : ${ }^{\star \star} ; p<0.01$ : ${ }^{\star \star} ; p<0.05$ : ${ }^{\star} ; p \geq 0.05$ : ns. The source data underlying Fig. 2a-e are provided as a Source Data file.

a

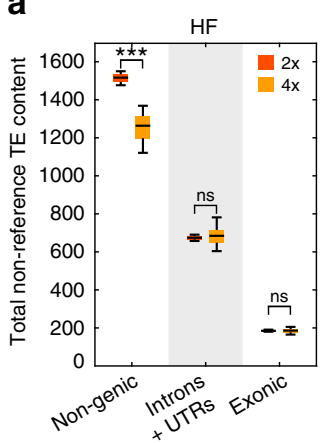

b

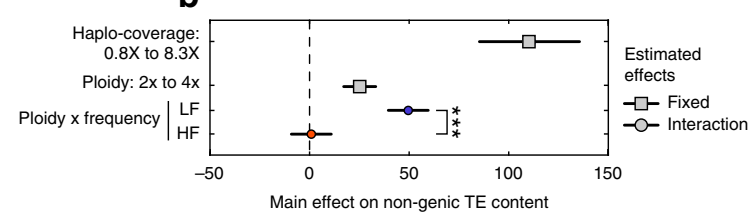

C

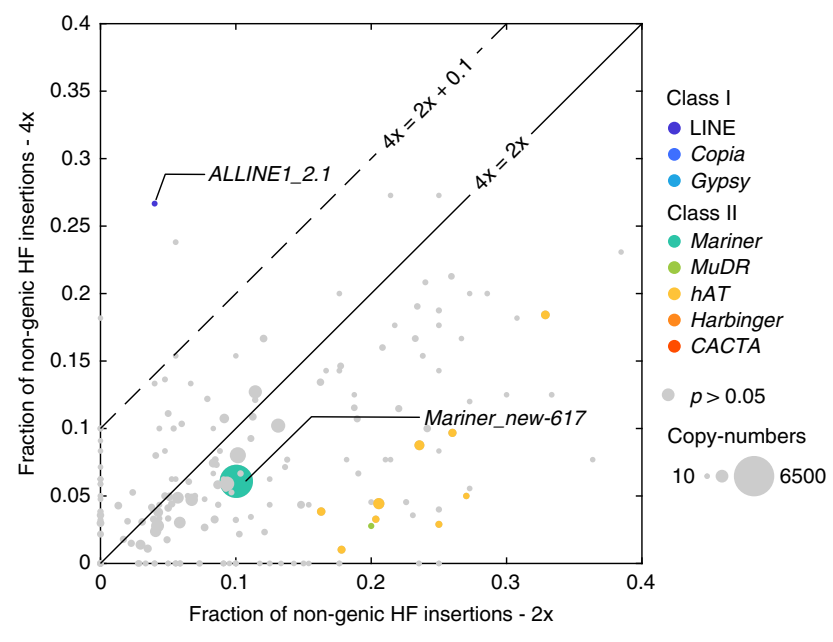

Fig. 3 Absence of genome-wide or family specific transposition burst hallmarks in autotetraploids. a Number of TE insertions carried by 100 individuals by category (non-genic, introns and UTRs, exonic) in diploids and tetraploids at high-frequency (HF) with standard deviations across 100 random samples and $p$-value of $t$-test between ploidies. Boxplot center lines, median; box limits, upper and lower quartiles; whiskers, 9th and 91st quantiles. b Estimated MLM effects and interaction effects of haplo-coverage, ploidy, and insertion frequency on non-genic TE content. Horizontal lines indicate $95 \%$ confidence intervals for each effect value. $p$-values for each coefficient corresponds to the $t$-statistic of the hypothesis test that the corresponding coefficient is equal to zero or not. c Fraction of non-genic insertions at high-frequency (HF) in tetraploids versus diploids by TE family. TE families with $\chi^{2} p$-values $<0.05$ are colored. $p<0.001$ : ${ }^{\star \star} ; p<0.01:{ }^{\star \star} ; p<0.05:{ }^{\star} ; p \geq 0.05$ : ns. The source data underlying Figs. 3 a and $3 c$ are provided as a Source Data file.

late flowering individual ${ }^{33,34}$, revealed a number of intergenic or intronic structural variants. Notably, two clones contained the same ATCOPIA78 solo-LTR insertion in the 2nd exon of AaFLC1 (Fig. $5 \mathrm{~d}$ ), which is the main contributor $(>80 \%)$ to total FLC expression in $A$. arenosa ${ }^{20}$. Examination of whole-genome sequencing data of the $286 \mathrm{~A}$. arenosa accessions for the presence of this solo-LTR insertion (see Methods) indicated that it is present in the three railway tetraploid populations, but not in any of the 15 diploid populations nor in any of the other 19 tetraploid populations analyzed (Fig. 5e). It was also absent from a hybrid mountain-railway population, which, unlike the other railway tetraploids, is characterized by high FLC expression and was shown previously to be early flowering because of a specific allele of CONSTANS, another major flowering-time regulator ${ }^{21}$. Although further work is required to prove the functional impact of the Copia insertion, the complete association with the loss of FLC expression supports the notion that Copia retrotransposons generate alleles that can enable rapid adaptation to novel habitats.

The ability of tetraploids to absorb locally adaptive variants through introgression with local diploids has been often argued to favor their potential for rapid adaptation 22,35 . At the TE level, we could confirm the strong admixture between southern Carpathians diploids and tetraploids (Supplementary Fig. 5a). Yet, even in this case, diploid TE insertions represented only a minor fraction of TE insertions at local high-frequency (Supplementary Fig. 5b) which are, in the case of Copia in particular, almost exclusively locally sourced within the south Carpathian tetraploids.

\section{Discussion}

Here, we performed a comprehensive analysis of TE dynamics in a natural diploid-autopolyploid species, which enabled us to assess the consequences of WGD without the confounding effect of hybridization. Our results indicate a major over-accumulation of TEs specifically within or near genes in the autotetraploids compared to their diploids counterparts, which supports the hypothesis that polysomy shelters TE insertions from selection. Conversely, we found no evidence of transposition bursts, neither genome-wide nor family-specific that could have been associated with the ancestral WGD event, which suggests that genome duplication alone, when not coupled with hybridization as in 

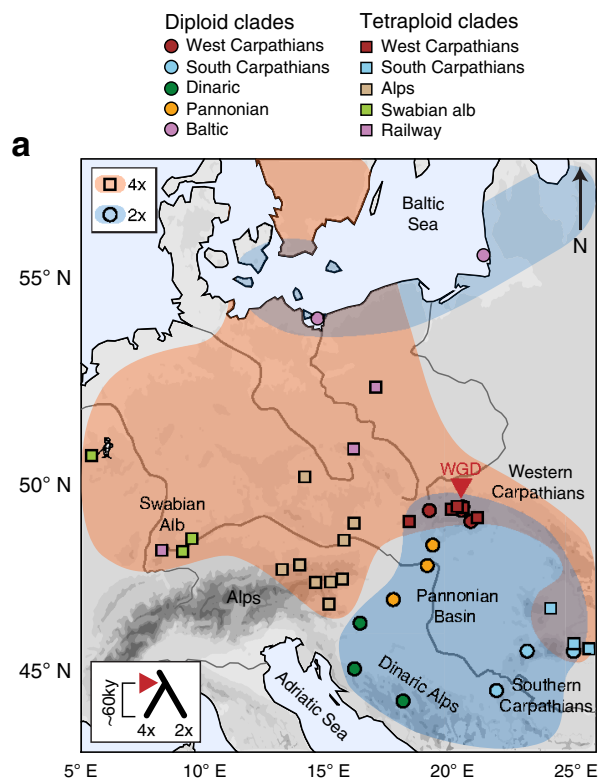

d Shared clade-specific insertions

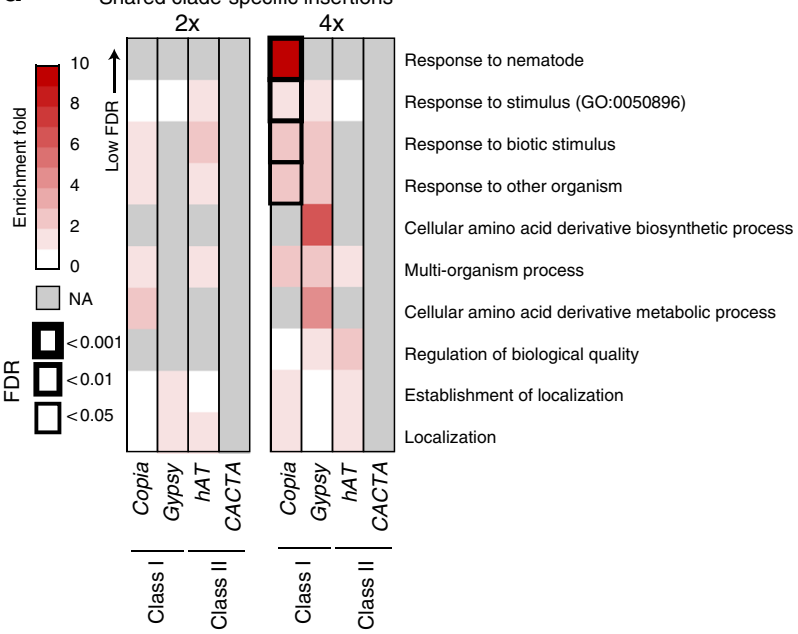

b

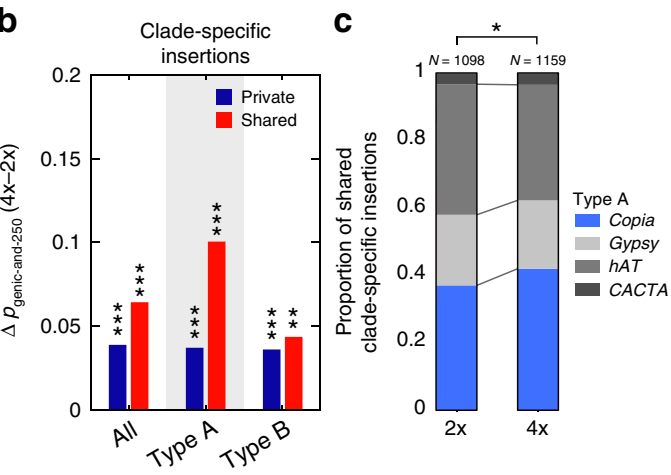

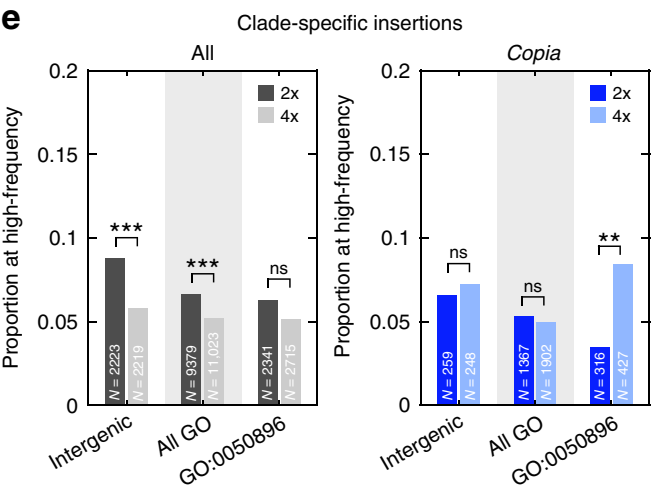

Fig. 4 Local positive selection on Copia genic insertions in tetraploids. a Map of the tetraploid and diploid populations colored by clade. $\mathbf{b}$ Difference between tetraploids and diploids of the proportion of genic and near-genic $(<250 \mathrm{bp}$ ) insertions present in only 1 clade (clade-specific) and private or shared within the clade for all, type A, and type B superfamilies with $p$-values of $\chi^{2}$ test between ploidies (type A: $2 \times n=4597$ private and 1098 shared, $4 x$ $n=5524$ private and 1159 shared; type B: $2 \times n=8921$ private and 2563 shared, $4 \times n=9646$ private and 2335 shared). c Proportion among shared cladespecific type $A$ insertions from each type A superfamily in diploids and tetraploids with $p$-value of $\chi^{2}$ test between ploidies. $\mathbf{d}$ GO enrichments in diploids and tetraploids among genes carrying or nearby shared clade-specific insertions for type A TE superfamilies. e Proportion in diploids and tetraploids of clade-specific insertions at locally high frequencies (shared by three or more individuals) within intergenic regions, within or near genes, or within or near stimulus response genes (GO:0050896) for all superfamilies or only Copia insertions with $p$-values of $\chi^{2}$ test between ploidies. $p<0.001$ : ${ }^{\star \star} ; p<0.01$ : $\star \star ; p<0.05:{ }^{\star} ; p \geq 0.05$ : ns. The source data underlying Fig. $4 \mathrm{~b}-\mathrm{e}$ are provided as a Source Data file.

allopolyploids (e.g. tobacco ${ }^{36}$, rapeseed $^{37}$ ), is insufficient to cause a severe "genomic stress" 38 . Finally, we present data supporting a role in local adaptation of the genic TE variants accumulating in tetraploids, including within a major adaptive locus.

Our conclusion that purifying selection in autotetraploids is relaxed confirms previous work based on single nucleotide polymorphisms (SNPs) ${ }^{22}$. However, given the young age of $A$. arenosa tetraploids ${ }^{16}$, the increase in mutational load generated by SNPs remains subtle 22 whereas the impact of relaxing purifying selection is magnified for TE insertions, which often produce major-effect alleles contrary to SNPs. Over the long term, we expect this fundamental difference to be further exacerbated in a chain reaction-like manner as has been observed in A. thaliana TE-mutation-accumulation lines ${ }^{30}$. In fact, transposition rates should rapidly increase in tetraploid genomes as more active copies are tolerated and intergenic space gradually widens, increasing further the genome tolerance for more transposition events.
In turn, the progressive over-accumulation of genic TE variants caused by relaxed purifying selection could provide raw material for local adaptation of some tetraploid populations. Indeed, Copia insertions within or near stimulus response genes appear to be under local positive selection specifically in tetraploids. In contrast, interploidy admixture with local diploid populations contributes very few potentially adaptive Copia insertions.

The successful invasion of railway ballasts by $A$. arenosa tetraploids has been particularly intriguing as it represents a textbook example of the increased invasion potential often associated with polyploidy ${ }^{27-29,39}$, yet appears to rely, at least in part, on diploid alleles imported through admixture ${ }^{21}$. Here, however, we found that a tetraploid-specific Copia exonic insertion within AaFLC1 was exclusive to and shared by all of the rapid-cycling railway populations, with the exception of one hybrid population where early flowering was not associated with a loss of FLC expression ${ }^{21}$. Further work is needed to characterize case by case the phenotypic impact of local TE insertions in particular at the FLC locus. 
a

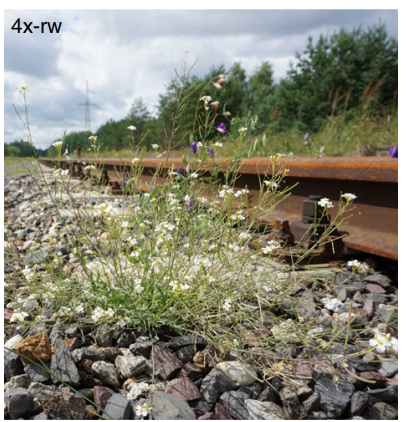

b

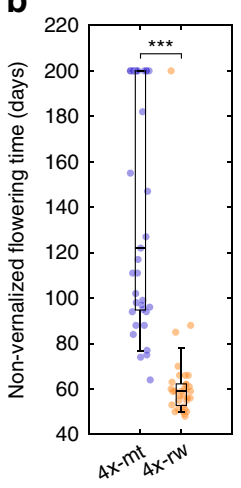

C

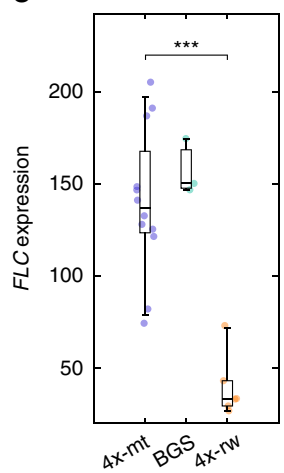

d

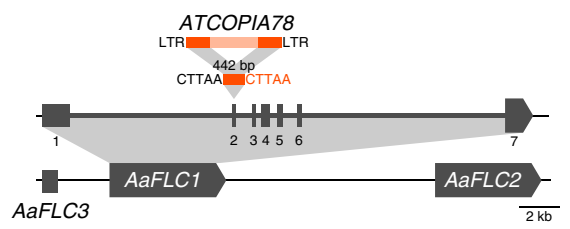

e

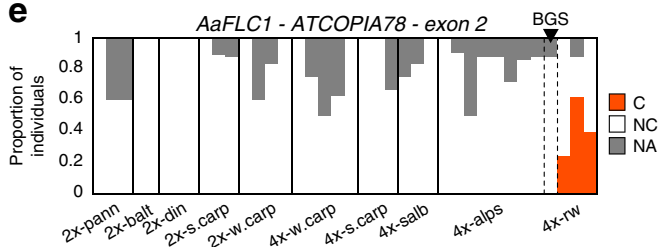

Fig. 5 Railway-specific ATCOPIA78 solo-LTR exonic insertion within FLC. a Picture of a tetraploid $A$. arenosa individual growing on railway ballasts near Świnoujście, Poland, in July 2017. b Non-vernalized flowering time, measured in days to the first open flower, of mountain (4x-mt) and railway (4x-rw) tetraploid populations. Data was redrawn from Baduel et al. ${ }^{20}$. Boxplot center lines, median; box limits, upper and lower quartiles; whiskers, 9th and 91st quantiles. c FLC expression tetraploid populations from mountain $(4 x-m t)$ and railway $(4 x-r w)$ and the hybrid mountain-railway population BGS. Data were redrawn from Baduel et al. ${ }^{35}$. Boxplot center lines, median; box limits, upper and lower quartiles; whiskers, 9th and 91st quantiles. $\mathbf{d}$ Map of FLC region with $442 \mathrm{bp}$-long ATCOPIA78 solo-LTR insertion in 2nd exon of AaFLC1 identified by fosmid sequencing. e Proportion of individuals across populations by clade (pann pannonian, balt baltic, din = dinaric, s.carp = south-carpathians, w.carp = west carpathians, salb $=$ swabian alb, rw $=$ railway) carrying $(C)$ or notcarrying (NC) the ATCOPIA78 solo-LTR insertion or not-assigned (NA). Hybrid mountain-railway population BGS is indicated with black triangle. The source data underlying Fig. $5 \mathrm{e}$ are provided as a Source Data file.

While TEs may provide adaptive opportunities, as examples in $A$. thaliana ${ }^{40}$ as well as in Drosophila ${ }^{41,42}$ suggest, their mobilization is likely to generate predominantly either neutral or deleterious alleles. In addition, TEs are major contributors of spurious recombination events", a key step in the "polyploid drop" 43 that has historically almost inevitably followed WGD events $^{3,44}$. Thus TE accumulation in autopolyploids may provide transient windows of adaptive opportunity but could eventually also lead to their evolutionary demise or at least their return to a diploid state.

In summary, our study sheds new light on the dynamic interactions between ploidy and the TE landscape with major implications for the adaptive potential and evolution of polyploids.

\section{Methods}

Data sources. Whole-genome sequencing data of $A$. arenosa individuals and paired-end alignments on the $A$. lyrata reference genome ${ }^{22,23}$ were obtained from Monnahan et al. ${ }^{22}$ where the phylogenetic relationships between the different clades of diploids and tetraploids (Fig. 4a) are described and analyzed.

Identification of non-reference TE insertions. Split and discordant reads were extracted from individual alignments $(2.8-18.1 \mathrm{X}$, average $8.4 \mathrm{X})$ and mapped on a joint TE library assembled from the annotation of all TEs in the A. lyrata reference genome $\left(87,477 \mathrm{TEs}^{26}\right)$. Mapped reads were then soft-clipped and re-mapped to the reference genome to define putative non-reference TE insertion sites. These sites were intersected across individuals to identify those shared and supported in at least one individual by a minimum of three reads, including at least one upstream and one downstream. Negative coverage, as defined by the minimum read depth over the upstream and downstream boundaries of a putative TE insertion site, was then calculated for each individual across all putative TE insertion sites. To limit false-negatives, non-carrier individuals with less than five reads negative coverage or more than $100(>10$ times the average read depth) were considered as missing information or NA. Sites with more than 10 NA diploids and $10 \mathrm{NA}$ tetraploids were considered non-informative for further analyses, resulting into 43,176 informative TE insertion sites (Supplementary Data 1). Due to the high heterozygosity of $A$. arenosa, in particular in tetraploids, we were not able to distinguish between homozygous and heterozygous TE insertions based on positive and negative coverage. Common TE names were obtained by intersection with TE annotation available in Pietzenuk et al. ${ }^{45}$ (MOESM3), or indicated as superfamily_new-followed by a unique identifier when previously unannotated (e.g. Mariner_new-617). Multiple TEs with the same common name were distinguished by a numbered suffix (e.g. ALLINE1_2.1).
Analyses of TE landscapes. Statistical analyses were performed using MATLAB (MathWorks, Inc., Natick, Massachusetts, United States). Carrier frequency was calculated at each TE insertion site over all non-NA individuals for the site. In diploids, $95 \%$ of TE insertions were at carrier-frequencies $(f)$ under $12.2 \%$. Under Hardy-Weinberg equilibrium, if $f$ is below $12.2 \%$, it should reflect allelic frequencies $(p)$ below $6.3 \%$ (from the formula $(1-p)^{2}=1-f$ ). Thus, $>97.8 \%$ of diploid carriers are expected to be heterozygous, i.e. $p \sim f / 2$. Similarly, in tetraploids at similar carrier-frequencies we should expect $>96.5 \%$ of carriers to be simplex heterozygotes, i.e. $p \sim f / 4$. This would suggest that at a given carrier frequency, corresponding allelic frequencies are lower in tetraploids than in diploids (by half). However, the coverage per haploid genome is also divided by 2 in tetraploids (thus ranging from $0.8 \mathrm{X}$ to $4.5 \mathrm{X}$ ), which reduces the sensitivity of detection of simplex heterozygotes especially at low frequencies. This bias would lead, on the opposite, to an underestimation of allelic frequencies from carrier-frequencies in tetraploids.

As we were not able to distinguish the zygosity of the carriers of TE insertions, and thus to establish unbiased estimates of allelic frequencies for both ploidies, we performed cross-ploidy comparisons based on carrier-frequencies instead. Indeed, the carrier-frequency spectrum (calculated over random samples of 100 individuals per ploidy corresponding to $1 \%$ frequency resolution) did not significantly differ between the two ploidies (Supplementary Fig. 3a). Carrier-frequency thresholds for LF and HF TE insertions were calculated as the $10 \%$ and $90 \%$ percentile of the diploid frequency spectrum $(\leq 1.2 \%$ vs $\geq 8.3 \%$, respectively), and the same thresholds were used in tetraploids.

Densities of TE insertions across chromosomes were calculated by $100 \mathrm{~kb}$ windows and smoothed using a LOWESS (Locally Weighted Scatterplot Smoothing) regression for plotting (Fig. 1c, d). Categories of TE insertions were based on the RNAseq-improved annotation of the A. lyrata reference genome 23,46 . In case of ambiguity the following priority order was given: $3^{\prime} \mathrm{UTR}-$ $5^{\prime}$ UTR-exon-intron- $<250$ bp upstream- $<250$ bp downstream. For TE insertions both $<2 \mathrm{~kb}$ upstream of one gene and $<2 \mathrm{~kb}$ downstream of another gene the attribution to one of the two categories was defined by the closest gene. The representations of these categories in the reference genome (WG coverage in Fig. 1h) were obtained following the same rules and were then normalized by their average coverage calculated in $10 \mathrm{~kb}$ windows using a high-coverage diploid alignment (47X). Pairwise differences in proportions by category (either LF vs HF or $2 \mathrm{x}$ vs $4 \mathrm{x}$ ) were estimated using a $2 \times 2 \chi^{2}$-test. Sequencing coverage is higher in tetraploids compared to diploids: $3.3-18.1 \mathrm{X}$ (average $8.7 \mathrm{X}$ ) versus $2.8-16.5 \mathrm{X}$ (average 7.9X), but lower when expressed per haploid genome (haplo-coverage). As most TE insertions are likely heterozygotes (see calculations above) the sensitivity of the SPLITREADER is expected to be lower in tetraploids compared to diploids. Therefore, multiple linear models (MLMs) of individual nonreference TE content (Figs. 2f-3b) were obtained by stepwise multiple linear regressions using as parameters haplo-coverage (average coverage by haploid genome), ploidy, and category of TE insertion (non-genic, exonic, and other genic) for Fig. 2f, or haplo-coverage, ploidy, and carrier frequency (LF and HF) for Fig. 3b (Supplementary Tables 1, 2). Fixed effects or interaction terms were added or removed based on the $p$-value for an $F$-test of the change in the sum of squared error with or without the term. Average TE content by ploidy was calculated based on 100 subsamples of 100 individuals by ploidy. Error-bars indicate standard deviation observed across 100 samples and statistical 
differences were obtained by 2 -way $t$-tests (Figs. $2 \mathrm{~g}$-3a). Gene Ontology (GO) enrichments were calculated based on genes $<250 \mathrm{bp}$ away from TE insertions of interest using agriGO in comparison with A. thaliana reference annotation (http://bioinfo.cau.edu.cn/agriGO/).

RNAseq analysis. RNAseq datasets were obtained from previously published datasets $^{21,22}$ for a subset of six tetraploid and three diploid populations with three individuals each. For each gene, we compared the average expression levels in populations where the nearest TE insertion was detected (carrier, $\mathrm{C}$ populations) to populations where the nearest TE insertion was not detected (non-carrier, NC). For genes with TE insertions $>2 \mathrm{~kb}$ away (intergenic) $\mathrm{C} / \mathrm{NC}$ ratio distribution was compared to randomly picked carriers and non-carriers populations (Supplementary Fig. 1a, Kolmogorov-Smirnov test). For genes with TE insertions $<250$ bp away (near-genic TE insertions), the distribution of $\mathrm{C} / \mathrm{NC}$ ratios was compared to both random (KS; $p<5 e-10)$ and intergenic (Supplementary Fig. $1 \mathrm{~b}, \mathrm{KS} p<5 \mathrm{e}-3$ ).

Fosmid libraries. Because the FLC region in A. arenosa contains $3 F L C$ paralogues (two entire copies AaFLC1 \& AaFLC2 and one truncated AaFLC3), that arose independently from the duplicated A. lyrata FLC paralogues ${ }^{33}$, Illumina shortreads align poorly on the FLC locus of A. lyrata reference genome impairing the detection of non-reference TE insertions. Instead, we extracted DNA from threeweek-old plants from a mainland railway population (TBG) using a large-scale CTAB protocol including treatment with pectinase. We constructed a fosmid library using the Copy Control Fosmid Library Production Kit (Epicentre) and screened it using DIG-labeled (Roche) PCR probes to the center of the FLC locus (primers 5' AGTGTAACTTCAATGGCAGAAAACCCT 3' and 5' ATGTGGCGG TAAGCAGAGATGACC $3^{\prime}$ ). We bar-coded positive clones and sequenced $100 \mathrm{bp}$ paired-end reads on an Illumina HiSeq 2000. We aligned FLC reads (which performed poorly in de novo assembly) to an A. arenosa BAC (GenBank accession no. FJ461780) using BWA. We identified insertions and deletions by targeted de novo alignment using Velvet ${ }^{47}$. The 442 bp-long insertion present in the second exon of AaFLC1 in two out of three fosmids bore a $94.5 \%$ similarity with the LTR sequence of ATCOPIA78 (RepeatMasker blast) in addition to a $5 \mathrm{bp}$ tandem site duplication (TSD), as expected from Copia insertions ${ }^{48}$.

We detected carrier individuals for this Copia insertion from whole-genome resequencing data by re-mapping paired-end reads to two versions of an updated $\mathrm{BAC}$ sequence ${ }^{20}$ including or not the TE insertion. We considered as carrier any individual with at least 1 read bridging the insertion extremities by at least $20 \mathrm{bp}$, and as non-carrier when no read bridged insertion extremities and at least 4 reads bridged the TSD.

Reporting summary. Further information on research design is available in the Nature Research Reporting Summary linked to this article.

\section{Data availability}

Whole-genome resequencing data of Monnahan et al. ${ }^{22}$ are deposited in the Sequence Read Archive with the primary accession code PRJNA484107 and PRJNA472485 for RNAseq data. Data supporting the findings of this work are available within the paper and its Supplementary Information files. A reporting summary for this Article is available as a Supplementary Information file. The datasets generated and analyzed during the current study are available from the corresponding authors upon request. The source data underlying Figs. 1b-h, 2a-e, 2g, 3a, 3c, 4b-e, and 5e, and Supplementary Figs. 1-5 are provided as a Source Data file.

\section{Code availability}

The SPLITREADER ${ }^{25}$ code is freely available at https://github.com/LeanQ/ SPLITREADER, Bowtie2 v2.3.2 at https://sourceforge.net/projects/bowtie-bio/files/ bowtie2/2.3.5.1/, Picard Tools at https://broadinstitute.github.io/picard/, SAMtools V1.2.1 at http://www.htslib.org/download/, and MATLAB version 9.3.0 (R2017b) from the MathWorks Inc. https://www.mathworks.com/.

Received: 24 June 2019; Accepted: 21 November 2019; Published online: 20 December 2019

\section{References}

1. Gregory, T. R. \& Ryan Gregory, T. in The Evolution of the Genome 3-87. https://doi.org/10.1016/b978-012301463-4/50003-6 (2005).

2. Wood, T. E. et al. The frequency of polyploid speciation in vascular plants. Proc. Natl Acad. Sci. USA 106, 13875-13879 (2009).

3. Wendel, J. F. The wondrous cycles of polyploidy in plants. Am. J. Bot. 102, 1753-1756 (2015).

4. Casacuberta, E. \& González, J. The impact of transposable elements in environmental adaptation. Mol. Ecol. 22, 1503-1517 (2013).
5. McClintock, B. The significance of responses of the genome to challenge. Science 226, 792-801 (1984)

6. Hegarty, M. J. et al. Transcriptome shock after interspecific hybridization in Senecio is ameliorated by genome duplication. Curr. Biol. 16, 1652-1659 (2006).

7. Parisod, C. et al. Rapid structural and epigenetic reorganization near transposable elements in hybrid and allopolyploid genomes in Spartina. New Phytol. 184, 1003-1015 (2009).

8. Ronfort, J. The mutation load under tetrasomic inheritance and its consequences for the evolution of the selfing rate in autotetraploid species. Genet. Res. 74, 31-42 (1999).

9. Vicient, C. M. \& Casacuberta, J. M. Impact of transposable elements on polyploid plant genomes. Ann. Bot. 120, 195-207 (2017).

10. Piednoël, M., Carrete-Vega, G. \& Renner, S. S. Characterization of the LTR retrotransposon repertoire of a plant clade of six diploid and one tetraploid species. Plant J. 75, 699-709 (2013).

11. Bardil, A., Tayalé, A. \& Parisod, C. Evolutionary dynamics of retrotransposons following autopolyploidy in the Buckler Mustard species complex. Plant J. 82, 621-631 (2015).

12. Kashkush, K., Feldman, M. \& Levy, A. A. Transcriptional activation of retrotransposons alters the expression of adjacent genes in wheat. Nat. Genet. 33, 102-106 (2003).

13. Zhang, J. et al. Autotetraploid rice methylome analysis reveals methylation variation of transposable elements and their effects on gene expression. Proc. Natl Acad. Sci. USA 112, E7022-E7029 (2015).

14. Otto, S. P. \& Goldstein, D. B. Recombination and the evolution of diploidy. Genetics 131, 745-751 (1992).

15. Otto, S. P. \& Whitton, J. Polyploid incidence and evolution. Annu. Rev. Genet. 34, 401-437 (2000)

16. Arnold, B., Kim, S.-T. \& Bomblies, K. Single geographic origin of a widespread autotetraploid Arabidopsis arenosa lineage followed by interploidy admixture. Mol. Biol. Evol. 32, 1382-1395 (2015).

17. Molina-Henao, Y. F. \& Hopkins, R. Autopolyploid lineage shows climatic niche expansion but not divergence in Arabidopsis arenosa. Am. J. Bot. 106, 61-70 (2019)

18. Brochmann, C. et al. Polyploidy in arctic plants. Biol. J. Linn. Soc. 82, 521-536 (2004).

19. Parisod, C., Holderegger, R. \& Brochmann, C. Evolutionary consequences of autopolyploidy. New Phytol. 186, 5-17 (2010).

20. Baduel, P., Arnold, B., Weisman, C. M., Hunter, B. \& Bomblies, K. Habitatassociated life history and stress-tolerance variation in Arabidopsis arenosa. Plant Physiol. 171, 437-451 (2016).

21. Baduel, P., Hunter, B., Yeola, S. \& Bomblies, K. Genetic basis and evolution of rapid cycling in railway populations of tetraploid Arabidopsis arenosa. PLoS Genet. 14, e1007510 (2018)

22. Monnahan, P. et al. Pervasive population genomic consequences of genome duplication in Arabidopsis arenosa. Nat. Ecol. Evol. 3, 457-468 (2019).

23. $\mathrm{Hu}, \mathrm{T}$. T. et al. The Arabidopsis lyrata genome sequence and the basis of rapid genome size change. Nat. Genet. 43, 476-481 (2011).

24. Hollister, J. D. et al. Genetic adaptation associated with genome-doubling in autotetraploid Arabidopsis arenosa. PLoS Genet. 8, e1003093 (2012).

25. Quadrana, L. et al. The Arabidopsis thaliana mobilome and its impact at the species level. Elife 5, e15716 (2016).

26. Legrand, S. et al. Differential retention of transposable element-derived sequences in outcrossing Arabidopsisgenomes. Mobile DNA. https://doi.org/ 10.1186/s13100-019-0171-6 (2019)

27. Soltis, P. S. \& Soltis, D. E. The role of genetic and genomic attributes in the success of polyploids. Proc. Natl Acad. Sci. USA 97, 7051-7057 (2000).

28. Pandit, M. K., Pocock, M. J. O. \& Kunin, W. E. Ploidy influences rarity and invasiveness in plants. J. Ecol. 99, 1108-1115 (2011)

29. Prentis, P. J., Wilson, J. R. U., Dormontt, E. E., Richardson, D. M. \& Lowe, A J. Adaptive evolution in invasive species. Trends Plant Sci. 13, 288-294 (2008)

30. Quadrana, L. et al. Transposition favors the generation of large effect mutations that may facilitate rapid adaption. Nat. Commun. 10, 3421 (2019).

31. Mondragón-Palomino, M., Meyers, B. C., Michelmore, R. W. \& Gaut, B. S. Patterns of positive selection in the complete NBS-LRR gene family of Arabidopsis thaliana. Genome Res 12, 1305-1315 (2002).

32. Meyers, B. C., Kaushik, S. \& Nandety, R. S. Evolving disease resistance genes Curr. Opin. Plant Biol. 8, 129-134 (2005).

33. Nah, G. \& Jeffrey Chen, Z. Tandem duplication of the FLC locus and the origin of a new gene in Arabidopsis related species and their functional implications in allopolyploids. New Phytol. 186, 228-238 (2010).

34. Wang, J., Tian, L., Lee, H.-S. \& Chen, Z. J. Nonadditive regulation of FRI and FLC loci mediates flowering-time variation in Arabidopsis allopolyploids. Genetics 173, 965-974 (2006)

35. Baduel, P., Bray, S., Vallejo-Marin, M., Kolář, F. \& Yant, L. The 'polyploid hop': shifting challenges and opportunities over the evolutionary lifespan of genome duplications. Front. Ecol. Evol. 6, https://doi.org/10.3389/ fevo.2018.00117 (2018). 
36. Petit, M. et al. Mobilization of retrotransposons in synthetic allotetraploid tobacco. New Phytol. 186, 135-147 (2010).

37. Sarilar, V. et al. Allopolyploidy has a moderate impact on restructuring at three contrasting transposable element insertion sites in resynthesized Brassica napus allotetraploids. New Phytol. 198, 593-604 (2013).

38. Parisod, C. \& Senerchia, N. in Plant Transposable Elements (eds Grandbastien, M. A. \& Casacuberta, J.) 147-168 (Springer, 2012).

39. Pandit, M. K., Tan, H. T. W. \& Bisht, M. S. Polyploidy in invasive plant species of Singapore. Bot. J. Linn. Soc. 151, 395-403 (2006)

40. Li, Z.-W. et al. Transposable elements contribute to the adaptation of Arabidopsis thaliana. Genome Biol. Evol. 10, 2140-2150 (2018).

41. González, J., Karasov, T. L., Messer, P. W. \& Petrov, D. A. Genome-wide patterns of adaptation to temperate environments associated with transposable elements in Drosophila. PLoS Genet. 6, el000905 (2010).

42. Lerat, E. et al. Population-specific dynamics and selection patterns of transposable element insertions in European natural populations. Mol. Ecol. 28, 1506-1522 (2019).

43. Mandáková, T. \& Lysak, M. A. Post-polyploid diploidization and diversification through dysploid changes. Curr. Opin. Plant Biol. 42, 55-65 (2018).

44. Soltis, P. S., Marchant, D. B., Van de Peer, Y. \& Soltis, D. E. Polyploidy and genome evolution in plants. Curr. Opin. Genet. Dev. 35, 119-125 (2015).

45. Pietzenuk, B. et al. Recurrent evolution of heat-responsiveness in Brassicaceae COPIA elements. Genome Biol. 17, 209 (2016).

46. Rawat, V. et al. Improving the annotation of Arabidopsis lyrata using RNASeq data. PLoS ONE 10, e0137391 (2015).

47. Zerbino, D. R. \& Birney, E. Velvet: algorithms for de novo short read assembly using de Bruijn graphs. Genome Res. 18, 821-829 (2008).

48. Berg, D. E. \& Howe, M. M. Mobile DNA (American Society for Microbiology, Washington, DC, 1989).

\section{Acknowledgements}

P.B. would like to thank Levi Yant for discussions and access to unpublished data, Vincent Castric for communication of unpublished A. lyrata TE annotation, and Pirita Paajanen for the high-coverage diploid A. arenosa alignment. Work in the Colot lab is supported by the Investissements d'Avenir ANR-10-LABX-54 MEMO LIFE, 506 ANR11-IDEX-0001-02 PSL* Research University. L.Q. acknowledges support from the MOMENTUM program of the Centre National de la Recherche Scientifique. P.B. was the recipient of a postdoctoral fellowship (code SPF20170938626) from the Fondation pour la Recherche Médicale (FRM). Further support was through a European Research Council Consolidator grant CoG EVO-MEIO 681946 to K.B.

\section{Author contributions}

P.B. designed the study with contributions from L.Q., K.B., and V.C. P.B. analyzed the whole genome resequencing data with help from L.Q. B.H. obtained and sequenced the fosmid clones. P.B. performed the assembly of the fosmid sequences as well as the identification and characterization of the ATCOPIA78 insertion. P.B. and V.C. wrote the manuscript. All authors revised the manuscript.

\section{Competing interests}

The authors declare no competing interests.

\section{Additional information}

Supplementary information is available for this paper at https://doi.org/10.1038/s41467019-13730-0.

Correspondence and requests for materials should be addressed to V.C.

Peer review information Nature Communications thanks Olivier Panaud, Christian Parisod, Hadi Quesneville and the other, anonymous, reviewer(s) for their contribution to the peer review of this work. Peer reviewer reports are available.

Reprints and permission information is available at http://www.nature.com/reprints

Publisher's note Springer Nature remains neutral with regard to jurisdictional claims in published maps and institutional affiliations.

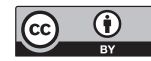

Open Access This article is licensed under a Creative Commons Attribution 4.0 International License, which permits use, sharing, adaptation, distribution and reproduction in any medium or format, as long as you give appropriate credit to the original author(s) and the source, provide a link to the Creative Commons license, and indicate if changes were made. The images or other third party material in this article are included in the article's Creative Commons license, unless indicated otherwise in a credit line to the material. If material is not included in the article's Creative Commons license and your intended use is not permitted by statutory regulation or exceeds the permitted use, you will need to obtain permission directly from the copyright holder. To view a copy of this license, visit http://creativecommons.org/ licenses/by/4.0/.

(C) The Author(s) 2019 